\title{
Experimental evaluation of optimum modulation formats for pico-cellular access networks based on resonant tunneling diodes
}

\author{
L. M. Pessoa ${ }^{1}$, M. C. Monteiro, M. R. Pereira' ${ }^{1}$, H. M. Salgado ${ }^{1}$, B. Romeira ${ }^{2}$ and J. M. L. Figueiredo ${ }^{2}$ \\ ${ }^{1}$ INESC TEC, Faculdade de Engenharia, Universidade do Porto, Portugal \\ ${ }^{2}$ Departamento de Física, CEOT, Universidade do Algarve, Portugal \\ email: luis.m.pessoa@inescporto.pt
}

\begin{abstract}
Pico-cell based access networks are increasingly seen as a promising solution for the growing mobility and bandwidth user demands. A recently developed resonant tunneling diode oscillator integrated with a photodetector and a laser diode (RTD-PD-LD), is being considered as a viable low cost pico-cell solution for radio-over-fiber based distributed antenna systems. In this paper we experimentally evaluate both the uplink and downlink error vector magnitude performance of the RTD-PD-LD for different phase and frequency modulation formats. We conclude that frequency modulated formats achieve better performance in both uplink and downlink configurations, yet, Gaussian Minimum Shift Keying format also appears to be a practical alternative.
\end{abstract}

Keywords: Injection-locked oscillators, microwave photonics, resonant tunnelling diode, radio-over-fiber, modulation formats.

\section{INTRODUCTION}

In the last few years we have witnessed a tremendous growth in the adoption of wireless communications in access networks associated with both Wi-Fi and cellular technologies, especially for bandwidth demanding applications such as video streaming. In order to cope with the demand, mobile operators have implemented infrastructure design considerations such as increasing the number of base-stations, reducing their coverage area and improving their geographic distribution, which represent, depending on their coverage, micro, pico and femto-cells. Radio-over-fiber (RoF) technology is considered as a promising solution for the problem of high cost, energy consumption and complexity required for dense base-station deployments [1]. Furthermore, the constant evolution in wireless standards leads to high investments by operators every time they need to replace the access points to support a new technology. RoF is a clear alternative since the complexity is shifted to the central station, which ensures a transparent base-station in terms of carrier frequency and protocols, and protects the investment of mobile operators [2]. Having in mind the necessity of simple and low cost solutions for future pico-cellular access networks, we have been developing innovative optoelectronic integrated circuits that could fulfil the RoF technology paradigm. These devices are based on a resonant tunnelling diode (RTD) optoelectronic oscillator (OEO) that takes advantage of a highly nonlinear current-voltage $(I-V)$ characteristic with a wide bandwidth negative differential resistance (NDR) region, inset of Fig. 1(a), that provides electrical gain to the circuit, and therefore can be used to produce self-sustained oscillations at very high frequencies (from $\mathrm{GHz}$ up to $\mathrm{THz}[3])$. The RTD-OEO analysed here, relies on a monolithic integration of a double barrier quantum well within a ridge waveguide photo-detector (PD) operating at $1.55 \mu \mathrm{m}$, which works as a RTD-PD oscillator (Fig. 1), and can be coupled to a single mode fibre (SMF). When optically or electrically injection locked, RTD based oscillators synchronize the frequency and phase of the free-running signal to the external injected RF signal, synchronizing to its phase modulation, which is very attractive in both microwave photonics and optical transmission systems [3,4,5]. When optically injected, it locks to the phase of the electrical carrier modulated on the received optical signal, performing optical to electrical (RF) conversion (downlink function). Conversely, when electrically injected it locks to the incoming RF signal and subsequently drives a laser diode (RTD-PD-LD), which is modulated with the synchronized radio frequency (RF) subcarrier (for the uplink function). The advantage of this concept relies in the integration of both microwave and optical functions in a single chip, which brings not only cost reduction but also increased speed and reliability.

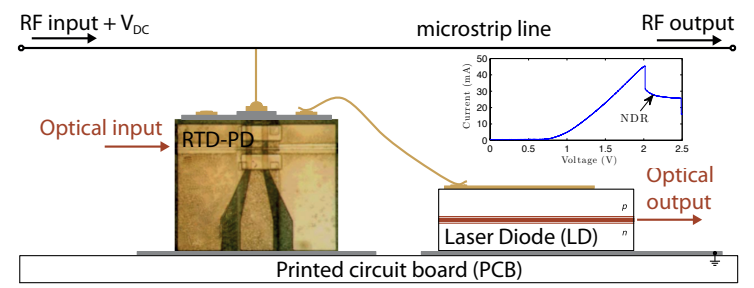

(a)

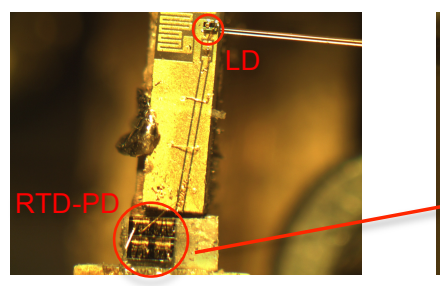

(b)

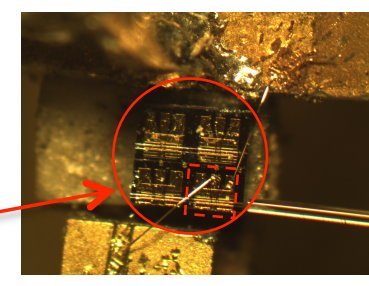

(c)

Fig. 1. (a) RTD-PD-LD schematic showing electrical and optical input and output ports, (b) microscope photography of the RTD-PD-LD prototype, with a SMF aligned with the LD, (c) detail of the RTD-PD having a SMF aligned with the optical waveguide (4 devices can be seen, but only the one in the dashed square is used). 
Although it is known that the RTD-PD-LD is capable of synchronizing to the phase modulation of an incoming electrical or optical signal, it remains to be determined what modulation formats provide optimum performance. In this work we demonstrate both uplink and downlink functional modes, through electrical and optical injection locking setups, respectively, and assess the error vector magnitude performance of the RTD-PD-LD for different phase and frequency modulation formats, namely phase shift keying (PSK), Gaussian minimum shift keying (GMSK) and frequency shift keying (FSK).

The paper is organized as follows: section 2 describes the experimental setup and measured results for the uplink function, section 3 provides the experimental setup and measured results related with the downlink function and finally the conclusions are given in section 4.

\section{UPLINK FUNCTION}

\subsection{Experimental Setup}

The electrical injection setup consisting of an electrical input and optical output signal is shown schematically in Fig. 2. We used a vector signal generator (VSG) to modulate a RF carrier with different modulation formats, which were injected into the RTD-LD through a customised Bias-T, in order to provide the DC biasing required for producing stable oscillation. The LD device employed here was a commercial (CST Global Ltd.) prototype operating around $1550 \mathrm{~nm}$ and $6 \mathrm{~mA}$ current threshold.

The laser optical output was coupled into a single mode fibre and converted to the electrical domain using a commercial photo-diode. The electrical output of the photo-diode was connected to a spectrum analyser with capability for performing signal down-conversion for signals with up to $8 \mathrm{MHz}$ bandwidth. The down-converted signal is then demodulated by Agilent 89600 vector signal analysis software running in a laptop, which is capable of determining the error vector magnitude (EVM) of the incoming signal.

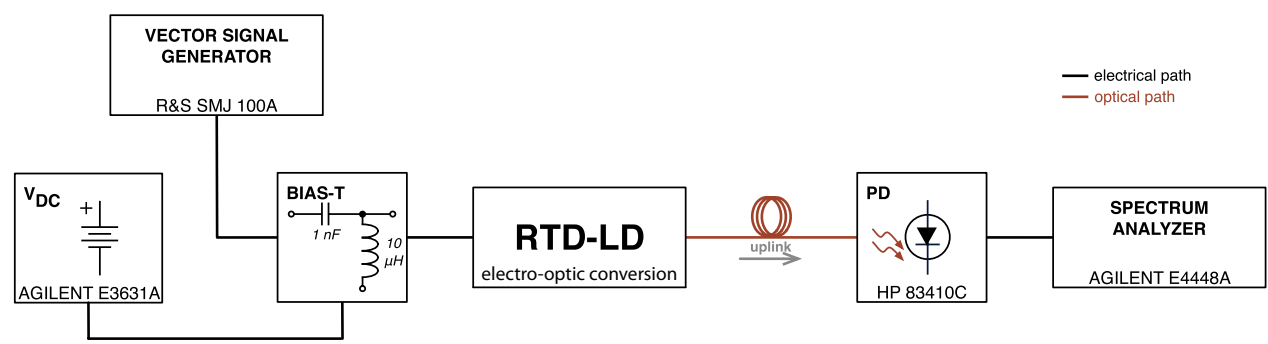

Fig. 2. Description of the uplink experimental setup

\subsection{Measurement results}

The device was found to oscillate between $700 \mathrm{MHz}$ and $1.2 \mathrm{GHz}$ for DC biasing ranging from $2.03 \mathrm{~V}$ up to 2.49 $\mathrm{V}$, respectively, producing a modulated optical signal at its LD output. The frequency oscillation upper limit was mainly limited by the gold wire length connections. The locking range was measured as a function of the injected electrical carrier power. It was found to be in average $\{8,18,35\} \mathrm{MHz}$, for injected powers of $\{-25,-15$, $-10\} \mathrm{dBm}$, respectively, which shows that the higher the injected power the larger the locking range. The experiments using a modulated injected electrical carrier were performed using a DC bias of $2.27 \mathrm{~V}$ which corresponds to a stable point of operation, where the device oscillates near $1.1 \mathrm{GHz}$, and provides a RF output power of $-10.7 \mathrm{dBm}$, measured after the photo-diode. The measured performance for different modulation formats is shown in Fig. 3, where the EVM was obtained as a function of the power of the injected electrical carrier, using a data-rate of 2 Msymbols/s.

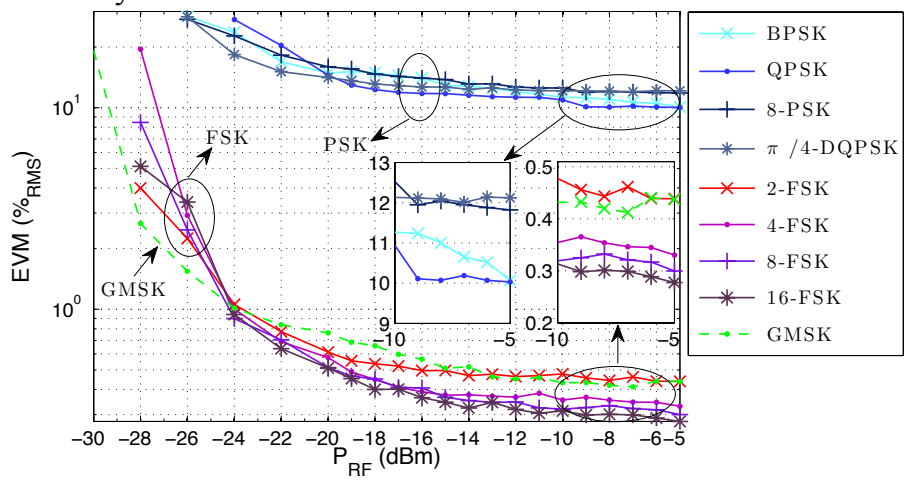

Fig. 3. EVM performance comparison for PSK, FSK and GMSK modulated formats for the uplink 
The results show that, as long as the injected power is higher than $-10 \mathrm{dBm}$, PSK modulations provide EVM measurements between $10 \%$ and $13 \%$, while for FSK and GMSK modulations the EVM varies between $0.25 \%$ and $0.5 \%$, which is one order of magnitude better. Furthermore, for PSK formats, the increase in the number of modulation levels leads to worse performance (except for BPSK and QPSK which have the same number of states per dimension), due to signal to noise ratio (SNR) increased requirements while the bandwidth remains constant. However, for FSK formats, the increase in the number of modulation levels leads to better performance due to the increase in occupied bandwidth, as clearly detailed in the inset of Fig. 3.

\section{DOWNLINK FUNCTION}

\subsection{Experimental Setup}

The optical injection setup consisting of an optical input and electrical output signals is shown schematically in Fig. 4. The VSG signal was used to modulate an optical carrier generated by a tuneable laser diode at $1550 \mathrm{~nm}$ using a $10 \mathrm{~Gb} / \mathrm{s}$ Mach-Zehnder modulator. The modulated optical signal is subsequently amplified by an erbium doped fibre amplifier (EDFA), then fed to a variable optical attenuator, and then split in a 90/10 coupler. One of the coupler outputs went to an optical multimeter used for controlling the power level. The other part of the optical signal was coupled to the RTD-PD ridge waveguide, which locks to the carrier modulated on the incoming optical signal and converts it to the electrical domain, with responsivity around 0.25 A/W [7]. Finally, a power supply provides the necessary DC biasing and a spectrum analyser downconverts the received signal to be demodulated by the Agilent 89600 VSA software.

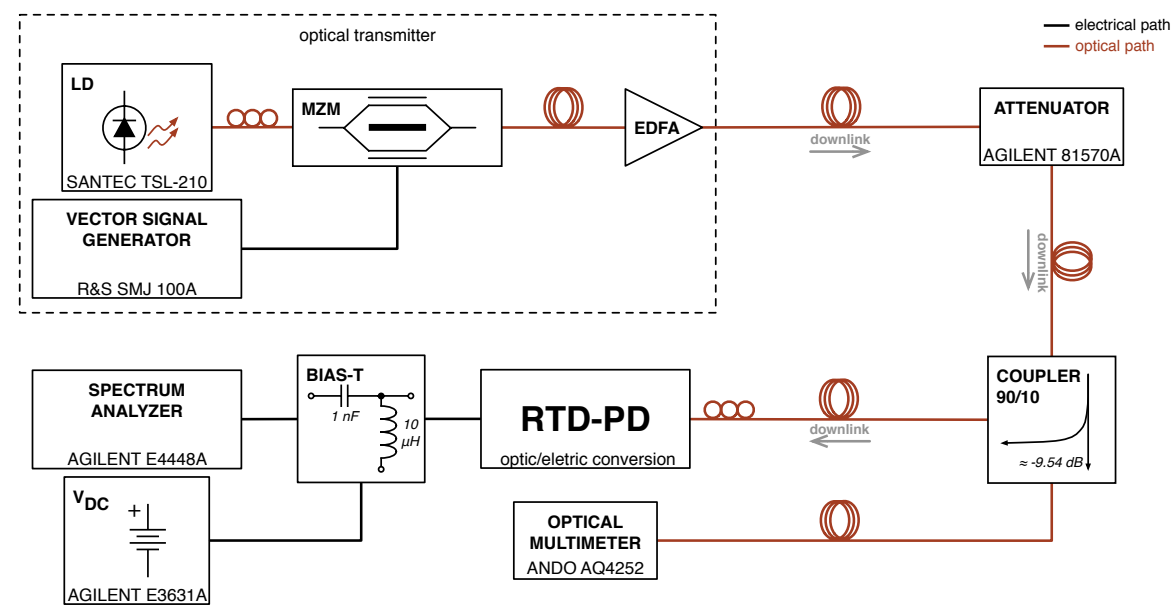

Fig. 4. Description of the downlink experimental setup

\subsection{Measurement results}

The locking range was measured as a function of the injected optical carrier power. It was found to be in average $\{2,4.2,6.5,9\} \mathrm{MHz}$, for injected optical powers of $\{-0.5,3.5,6.5,9.5\} \mathrm{dBm}$, respectively. The power of the electrical carrier on the VSG was kept at $17 \mathrm{dBm}$, and we verified that the locking range also depends significantly on this parameter. The results show that the higher the power of the modulating electrical carrier or the injected optical carrier the larger the locking range. The measured electrical output power of the RTD-OEO was $-7.9 \mathrm{dBm}$.

The measured performance for different modulation formats is shown in Fig. 5, where the EVM was obtained as a function of the power of the electrical carrier modulation, using a data-rate of 2 Msymbols/s, for a fixed injected optical power of $9.5 \mathrm{dBm}$. The PSK modulation formats provide EVM results no better than 14\%, while FSK based formats achieve $0.55 \%$ and GMSK attains $1 \%$ in the best case, which is again one order of magnitude better. While GMSK achieved a similar performance compared to 2-FSK in the uplink scenario, this does not hold true for the downlink, where GMSK performs slightly worse. It can be observed that the downlink performance is generally worse than that of the uplink. This can be explained by the limitations associated to the optical injection process itself, since the coupling method is still very incipient, which could be optimized in a future prototype, for example by including facets with anti-reflection coatings, or tapering the ridge end region. The poor efficiency of the optical coupling lead us to the necessity of amplifying the optical signal, which introduced noise and consequently degraded the EVM results. Furthermore, there is an observable performance 
degradation for electrical modulated powers above $10 \mathrm{dBm}$, which results from the nonlinearity in the MachZehnder modulator.

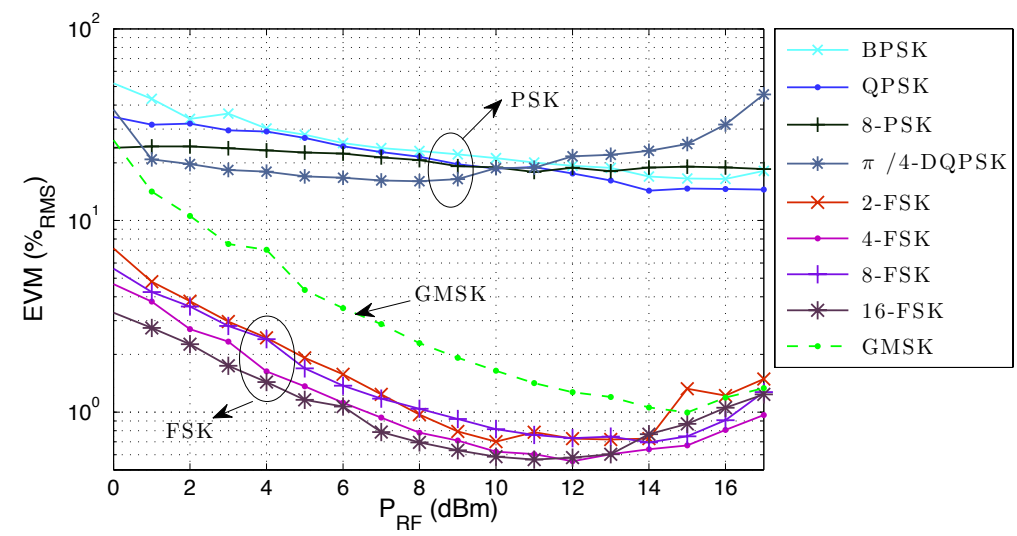

Fig. 5. EVM performance comparison for PSK, FSK and GMSK modulated formats for the downlink

\section{CONCLUSIONS}

We have determined the error vector magnitude performance of different phase and frequency modulation formats for both uplink and downlink functions using RTD-based optoelectronic integrated circuits. We conclude that frequency modulated formats achieve better performance, although the Gaussian Minimum Shift Keying format also appears to be a practical alternative. Furthermore, future activities may address the performance of the device under realistic simultaneous uplink and downlink traffic, as well as the optimization of the optical injection efficiency.

\section{ACKNOWLEDGMENT}

This work was supported by FCT under the project WOWi (PTDC/EEA-TEL/100755/2008) and in the framework of programme POCTI/FEDER with grant REEQ/1272/EEI/2005. B. Romeira and J. M. L. Figueiredo acknowledge the fruitful discussions with Professor Charles N. Ironside, Dr. A. E. Kelly, and Dr. H. Cantú of the School of Engineering, University of Glasgow, UK, on RTD optoelectronic oscillators.

\section{REFERENCES}

[1] A. Nirmalathas, C. Lim, and D. Novak, "Progress in millimeter-wave fiber-radio access networks," Annals of Telecommunications, vol. 56, no.1-2, pp. 27-38, 2001.

[2] P. P. Smyth, "Optical Radio - A Review of a Radical New Technology for Wireless Access Infrastructure," BT Technology Journal, vol. 21, no. 3, pp. 22-31, Aug. 2003.

[3] M. Feiginov, C. Sydlo, O. Cojocari, and P. Meissner, "Resonant-tunnelling-diode oscillators operating at frequencies above 1.1 THz," Appl. Phys. Lett., vol. 99, p. 233506, 2011.

[4] B. Romeira, J. Figueiredo, T. J. Slight, L. Wang, E. Wasige, and C. Ironside, "Wireless/photonics interfaces based on resonant tunneling diode optoelectronic oscillators," in Conference on Lasers and Electro-Optics \& Conference on Quantum electronics and Laser Science Conference, CLEO/QELS'09, Junho 2009, pp. 1-2. [5] H. Cantu, B. Romeira, K. Seunarine, A. Kelly, C. Ironside, and J. Figueiredo, "Conversion of Phase Modulated Signals from Optical Network to Wireless Domain using Resonant Tunneling Diode Optoelectronic Integrated Circuits," in National Fiber Optic Engineers Conference, NFOEC'12, California. Optical Society of America, Mar. 2012, p. JTh2A.19.

[6] B. Romeira, L.M. Pessoa, H.M. Salgado, S. Silva, and J.M.L. Figueiredo, "Clock recovery of an injectionlocked resonant tunneling diode microwave-photonics oscillator, " in Transparent Optical Networks (ICTON), 2011 13th International Conference on, pages 1-4. IEEE, 2011

[7] Romeira, B.; Figueiredo, J. M L; Ironside, C.N.; Kelly, A.E.; Slight, T.J., "Optical Control of a Resonant Tunneling Diode Microwave-Photonic Oscillator," Photonics Technology Letters, IEEE, vol.22, no.21, pp.16101612, Nov.1, 2010 